\title{
Author's response to letter on number needed to treat in COPD: exacerbations versus pneumonias
}

I thank Keene et al for their letter. ${ }^{1}$ I am pleased to clarify the issues they have raised about my paper. ${ }^{2}$ First, I agree that when dealing with recurrent events such as exacerbations, it is statistically more informative to analyse all events with tools such as incidence rates and rate differences. The point of my paper, however, was that inverting these rate differences and calling the result a number needed to treat (NNT) is simply wrong. For example, while the rates of chronic obstructive pulmonary disease (COPD) exacerbation from the TORCH trial are valid representations of the incidence, using them to compute an 'NNT of 4 to prevent one exacerbation in 1 year' is incorrect. ${ }^{2}$ The only valid NNT in this case is instantaneous, namely 1250 patients need to be treated on a given day to prevent one exacerbation on that day. ${ }^{2}$ Incidentally, the issue of extra-Poisson variability is not relevant to the rate, only to its variance and CIs, which were not the object of the NNT discussion. ${ }^{3}$

Second, to stretch the NNT over a treatment period longer than a day, one must use the cumulative incidence and not the rate. This is best done by the KaplanMeier approach that provides the cumulative incidence of the first exacerbation. ${ }^{4}$ This use of the first exacerbation is a necessary limitation if one is resolute in computing the NNT. Besides, the statement 'if all patients experience at least one event, then the patient-based NNT is infinite' is incorrect. ${ }^{1}$ The Kaplan-Meier curve is definitely estimable in this case (no censored observations), providing finite values for the patient-based NNT.

Third, the INSPIRE trial is, on the contrary, most suited for the question at hand as it reflects two pragmatic alternative ways in which clinicians treat COPD patients. ${ }^{5}$ Its conclusion that 'pneumonia is much less frequent than exacerbation in COPD' can be misconstrued as fluticasone having less 
Table 1 Computation of rates of chronic obstructive pulmonary disease (COPD) exacerbations requiring health care use (HCU) and of pneumonias in the INSPIRE trial, along with rate differences comparing the fluticasone-salmeterol combination with tiotropium ${ }^{5}$

\begin{tabular}{lccc}
\hline & Fluticasone + salmeterol & Tiotropium & $\begin{array}{c}\text { Rate difference* } \\
\text { (per 100/year) }\end{array}$ \\
\hline Number of subjects & 658 & 665 & \\
Days on treatment (mean) & 561 & 519 & \\
Person-years on treatment (total) & 1011 & 945 & \\
Number of exacerbations (HCUt) & 1185 & 1070 & +4.0 \\
Rate per year (per 100) & 117.2 & 113.2 & \\
Number of pneumonias & 62 & 25 & +3.5 \\
Rate per year (per 100) & 6.1 & 2.6 & \\
\hline *Rate difference = rate for fluticasone-salmeterol combination minus rate for tiotropium. & & \\
tExacerbations requiring health care use. & &
\end{tabular}

impact on pneumonia than on exacerbations. ${ }^{6}$ This impact must be based on difference or ratio measures including the NNT. Using all 87 pneumonias and 2255 COPD exacerbations requiring healthcare from that trial, rather than only the first event, as table 1 displays, the rate differences show that the fluticasone-salmeterol combination increases the frequencies of COPD exacerbations, albeit not significantly, and of pneumonia compared with tiotropium.

While not particularly for or against the NNT measure of impact, I remain a strong advocate of clarity in the presentation and interpretation of scientific data, especially in journals with a clinical penchant.

\section{Samy Suissa}

Correspondence to Prof Samy Suissa, Department of Epidemiology and Biostatistics, Centre for Clinical Epidemiology, Lady Davis Institute, Jewish General Hospital, McGill University, 3755 Cote Ste-Catherine, H-461, Montréal, Québec, Canada H3T 1E2; samy.suissa@mcgill.ca

Competing interests The author has received research grants and/or participated in advisory meetings or as conference speaker for AstraZeneca, BoehringerIngelheim, Forest, GlaxoSmithKline, Merck, Novartis, Nycomed and Pfizer.

Provenance and peer review Not commissioned; internally peer reviewed.

To cite Suissa S. Thorax 2013;68:882-883.

Received 20 February 2013

Accepted 21 February 2013

Published Online First 22 March 2013

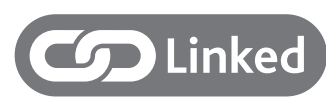

http://dx.doi.org/10.1136/thoraxjnl-2013-203403

Thorax 2013;68:882-883.

doi:10.1136/thoraxjnl-2013-203461

\section{REFERENCES}

1 Keene ON, Anzeuto A, Ferguson GT, et al. Number needed to treat in COPD: exacerbations versus pneumonias. Thorax 2013;68:883.
2 Suissa S. Number needed to treat in COPD: exacerbations versus pneumonias. Thorax 2013;68:540-3.

3 Suissa S. Statistical treatment of exacerbations in therapeutic trials of chronic obstructive pulmonary disease. Am J Respir Crit Care Med 2006;173:842-6.

4 Cates C. Inhaled corticosteroids in COPD: quantifying risks and benefits. Thorax 2013;68:499-500.

5 Wedzicha JA, Calverley PM, Seemungal TA, et al. The prevention of chronic obstructive pulmonary disease exacerbations by salmeterol/fluticasone propionate or tiotropium bromide. Am J Respir Crit Care Med 2008;177:19-26.

6 Calverley PM, Stockley RA, Seemungal TA, et al. Reported pneumonia in patients with COPD: findings from the INSPIRE study. Chest 2011;139:505-12. 